\title{
A GENERALIZATION OF QUASI-MONOTONE SEQUENCES
}

\author{
by M. M. ROBERTSON
}

(Received 27th April 1967)

\section{Introduction}

The object of this note is to generalize the notion of quasi-monotony for sequences of real numbers and to prove corresponding generalizations of certain known theorems. First, we recall the definition of quasi-monotony.

Definition 1. A sequence $\left\{a_{n}\right\}$ is said to be quasi-monotone if and only if $n^{-\beta} a_{n} \downarrow 0$ for some $\beta \geqq 0$ or equivalently if and only if $a_{n} \geqq 0$ and $\Delta a_{n} \geqq-\alpha n^{-1} a_{n}$ for some $\alpha \geqq 0$. (Here $\Delta a_{n}=a_{n}-a_{n+1}$ ).

The generalized notion referred to in the title is to be called $(\phi, \delta)$-monotony and this gives rise to

Definition 2. A sequence $\left\{a_{n}\right\}$ is said to be $(\phi, \delta)$-monotone if and only if $a_{n} \rightarrow 0, a_{n} \geqq 0$ ultimately and $\Delta a_{n} \geqq-\delta_{n+1}$, where the $\delta_{n}$ form a sequence of non-negative numbers, $\left\{\phi_{n}\right\}$ is a positive monotone increasing sequence and $\Sigma \phi_{n} \delta_{n}<\infty$.

It is easily seen that every monotone decreasing null sequence is $(\phi, \delta)$ monotone for all sequences $\left\{\phi_{n}\right\},\left\{\delta_{n}\right\}$ such that $\Sigma \phi_{n} \delta_{n}<\infty$. Also, we observe that a quasi-monotone sequence $\left\{a_{n}\right\}$ in which $a_{n} \rightarrow 0$ is $(\phi, \delta)$-monotone with $\delta_{n+1}=\alpha n^{-1} a_{n}$ for all sequences $\left\{\phi_{n}\right\}$ such that $\Sigma \phi_{n+1} n^{-1} a_{n}<\infty$. In a recent note, Boas (1) defined $\delta$-quasi-monotone sequences and obtained a number of theorems involving them. These results of Boas are concerned with the particular monotone increasing sequences $\left\{n^{\nu}\right\}(\gamma \geqq 0)$ and $\{\log n\}$ and, in our terminology, these $\delta$-quasi-monotone sequences are $\left(n^{\gamma}, \delta\right)$-monotone and $(\log n, \delta)$ monotone respectively. The following results on $(\phi, \delta)$-monotone sequences are first established in this paper.

Theorem 1. If the sequence $\left\{a_{n}\right\}$ is $(\phi, \delta)$-monotone and $\Sigma a_{n} \Delta \phi_{n}$ converges, then $a_{n} \phi_{n} \rightarrow 0$.

Theorem 2. If the sequence $\left\{a_{n}\right\}$ is $(\phi, \delta)$-monotone and $\Sigma a_{n} \Delta \phi_{n}$ converges, then the series $\Sigma \phi_{n+1} \Delta a_{n}$ is absolutely convergent.

Theorem 3. If $\left\{\lambda_{n}\right\}$ is a strictly increasing sequence of positive integers such that $\Delta \lambda_{n}=O\left(\Delta \lambda_{n-1}\right)$ as $n \rightarrow \infty$ and if the sequence $\left\{a_{n}\right\}$ is $(n, \delta)$-monotone, then the two series $\Sigma a_{n}$ and $\Sigma a_{\lambda_{n}} \Delta \lambda_{n}$ are either both convergent or both divergent.

The conclusion of Theorem 1 clearly holds for every convergent sequence $\left\{\phi_{n}\right\}$ and, as Theorem 2 is deduced from Theorem 1, this theorem is also true 
for convergent $\left\{\phi_{n}\right\}$. It is easily seen that Theorem 1 extends Olivier's theorem which shows that, for monotone decreasing null sequences $\left\{a_{n}\right\}, \Sigma a_{n}<\infty$ implies that $n a_{n} \rightarrow 0$. Theorem 3 provides an extension of the well known Cauchy condensation test for convergence. The extension of Cauchy's test for quasi-monotone sequences was proved by Shah (2) and Szász (4).

In order to state the next result, we require another definition.

Definition 3. A sequence $\left\{a_{n}\right\}$ is said to be $(\phi, \delta)$-positive if and only if it is the sequence of differences of $a(\phi, \delta)$-monotone sequence.

Theorem 4. If the sequence $\left\{a_{n}\right\}$ is $(\phi, \delta)$-positive and $\Sigma \phi_{n+1} a_{n}$ converges, then the series $\Sigma \phi_{n+1} a_{n}$ is absolutely convergent.

In the note of Boas mentioned above, several results were established concerning the integrability of trigonometric series. Here, we generalize two of these results to the case where the trigonometric sine or cosine coefficients form a $(\phi, \delta)$-monotone sequence. Previous results of this nature for quasi-monotone sequences were proved by Shah in (3). and

Theorem 5. Suppose that $\eta(x)$ is a non-negative function such that $\eta(x) \in L(0, \pi)$

$$
\phi_{n}=n \int_{0}^{1 / n} \eta(x) d x+\int_{1 / n}^{\pi} x^{-1} \eta(x) d x
$$

is a monotone increasing sequence. If $\left\{\lambda_{n}\right\}$ is a $(\phi, \delta)$-monotone sequence and $\Sigma \lambda_{n} \Delta \phi_{n}$ converges, then $\Sigma \lambda_{n} \cos n x$ is convergent for all values of $x$ (except perhaps at integral multiples of $2 \pi)$ and $\eta(x) \Sigma \lambda_{n} \cos n x \in L(0, \pi)$.

Theorem 6. Suppose that $\eta(x)$ is a non-negative function such that

and

$$
x \eta(x) \in L(0, \pi)
$$

$$
\phi_{n}=n^{2} \int_{0}^{1 / n} x \eta(x) d x+\int_{1 / n}^{\pi} x^{-1} \eta(x) d x
$$

is a monotone increasing sequence. If $\left\{\lambda_{n}\right\}$ is a $(\phi, \delta)$-monotone sequence and $\Sigma \lambda_{n} \Delta \phi_{n}$ converges, then $\Sigma \lambda_{n} \sin n x$ is convergent for all values of $x$ and

$$
\eta(x) \Sigma \lambda_{n} \sin n x \in L(0, \pi) \text {. }
$$

\section{Proofs of the first four theorems}

As has been already remarked, the conclusion of Theorem 1 is immediate when $\left\{\phi_{n}\right\}$ is convergent and so we assume that $\phi_{n} \uparrow \infty$. We note that

$$
\Delta\left(\phi_{r} a_{r}\right)=\phi_{r} a_{r}-\phi_{r+1} a_{r+1}=a_{r} \Delta \phi_{r}+\phi_{r+1} \Delta a_{r}
$$

and so, for $m<n$,

$$
\begin{aligned}
\phi_{m} a_{m}-\phi_{n} a_{n} & =\sum_{r=m}^{n-1} a_{r} \Delta \phi_{r}+\sum_{r=m}^{n-1} \phi_{r+1} \Delta a_{r} \\
& \geqq \sum_{r=m}^{n-1} a_{r} \Delta \phi_{r}-\sum_{r=m}^{n-1} \phi_{r+1} \delta_{r+1} .
\end{aligned}
$$


Since $\Sigma a_{n} \Delta \phi_{n}$ and $\Sigma \phi_{n} \delta_{n}$ converge, to every $\varepsilon>0$ there corresponds an integer $N$ such that

and therefore,

$$
\sum_{r=m}^{n-1} a_{r} \Delta \phi_{r}-\sum_{r=m}^{n-1} \phi_{r+1} \delta_{r+1} \geqq-\varepsilon
$$

$$
\phi_{m} a_{m}-\phi_{n} a_{n} \geqq-\varepsilon
$$

for all $m, n$ such that $N \leqq m<n$. Hence, since $\varepsilon$ is arbitrary, the sequence $\left\{\phi_{n} a_{n}\right\}$ can have at most one limit point. This limit point must be zero, as otherwise we shall show that a contradiction must occur.

Let us suppose that $\phi_{n} a_{n} \rightarrow l>0$. Then, for all sufficiently large $n, a_{n}>\frac{1}{2} l \phi_{n}^{-1}$ and so, since $\Sigma a_{n} \Delta \phi_{n}$ converges, $\Sigma \phi_{n}^{-1} \Delta \phi_{n}$ is convergent. Since the sequence $\left\{\phi_{n}\right\}$ is monotone increasing, we write $-c_{n}=\phi_{n}^{-1} \Delta \phi_{n}$. Then we have $c_{n} \geqq 0$, $\Sigma c_{n}$ convergent and $\phi_{n+1} / \phi_{n}=1+c_{n}$. Therefore,

$$
\lim _{n \rightarrow \infty} \phi_{n}=\phi_{1} \prod_{1}^{\infty}\left(1+c_{n}\right),
$$

which is the above mentioned contradiction since $\Pi\left(1+c_{n}\right)$ converges because of the convergence of $\Sigma c_{n}$ and $\phi_{n} \uparrow \infty$. This completes the proof.

To prove Theorem 2, we write $\left(\Delta a_{n}\right)^{-}=\max \left(0,-\Delta a_{n}\right)$ and obtain

$$
\phi_{n+1}\left|\Delta a_{n}\right|=\phi_{n+1} \Delta a_{n}+2 \phi_{n+1}\left(\Delta a_{n}\right)^{-}
$$

Since $0 \leqq\left(\Delta a_{n}\right)^{-} \leqq \delta_{n+1}$ and $\Sigma \phi_{n} \delta_{n}$ converges, we see that $\Sigma \phi_{n+1}\left(\Delta a_{n}\right)^{-}$is convergent. The proof is completed by showing that $\Sigma \phi_{n+1} \Delta a_{n}$ is convergent, and this follows by the Cauchy principle of convergence from (2.1) because both $\left\{\phi_{n} a_{n}\right\}$ and $\Sigma a_{n} \Delta \phi_{n}$ converge.

We now prove Theorem 3. First, we assume that $\Sigma a_{\lambda_{n}} \Delta \lambda_{n}$ is convergent. We have $\Delta a_{r} \geqq-\delta_{r+1}$ and so $a_{r+1} \leqq a_{r}+\delta_{r+1}$. It follows that

$$
a_{r} \leqq a_{\lambda_{n}}+\sum_{\lambda_{n}+1}^{r} \delta_{k}
$$

for $\lambda_{n}<r<\lambda_{n+1}$. This shows that

$$
\sum_{\lambda_{n}}^{\lambda_{n+1}-1} a_{r} \leqq\left(\lambda_{n+1}-\lambda_{n}\right) a_{\lambda_{n}}+\sum_{\lambda_{n}+1}^{\lambda_{n+1}-1} \delta_{k}\left(\lambda_{n+1}-k\right)
$$

and so, since

$$
\lambda_{n+1}-k<\lambda_{n+1}-\lambda_{n}<C\left(\lambda_{n}-\lambda_{n-1}\right)<C k
$$

for some fixed positive number $C$, we have

$$
\sum_{1}^{\infty} a_{n} \leqq-\sum_{1}^{\infty} a_{\lambda_{n}} \Delta \lambda_{n}+\sum_{1}^{\infty} n \delta_{n}
$$

Thus $\Sigma a_{n}$ converges since both $\Sigma a_{\lambda_{n}} \Delta \lambda_{n}$ and $\Sigma n \delta_{n}$ are convergent. The converse result is proved in a similar fashion.

Next we prove Theorem 4 . Since the sequence $\left\{a_{n}\right\}$ is $(\phi, \delta)$-positive, there 
is a $(\phi, \delta)$-monotone sequence $\left\{A_{n}\right\}$ such that $a_{n}=\Delta A_{n}=A_{n}-A_{n+1}$. Also, $A_{n} \rightarrow 0$ and so $\Sigma a_{n}$ is convergent and $A_{n}=\sum_{n}^{\infty} a_{k}$. Then the proof follows from Theorem 2 if we show that $\Sigma A_{n} \Delta \phi_{n}$ is convergent.

Now, there is an integer $N$ such that $A_{n} \geqq 0$ for all $n>N$ so that, in order to show that $\Sigma A_{n} \Delta \phi_{n}$ converges, we have only to show that $-\sum_{N+1}^{m} A_{n} \Delta \phi_{n}$ is bounded above for all $m>N$. We have

$$
-\sum_{N+1}^{m} A_{n} \Delta \phi_{n}=-\sum_{n=N+1}^{m} \Delta \phi_{n} \sum_{k=n}^{\infty} a_{k} \leqq-\sum_{n=N+1}^{m} \Delta \phi_{n} \sum_{k=n}^{\infty}\left(a_{k}+\delta_{k+1}\right)
$$

for all $m>N$. Since $a_{k}+\delta_{k+1} \geqq 0$ for all $k$, we can reverse the order of summation and obtain

$$
\begin{aligned}
&-\sum_{n=N+1}^{m} \Delta \phi_{n} \sum_{k=n}^{\infty}\left(a_{k}+\delta_{k+1}\right) \\
&=-\sum_{k=N+1}^{m} \sum_{n=N+1}^{k} \Delta \phi_{n}\left(a_{k}+\delta_{k+1}\right)-\sum_{k=m+1}^{\infty} \sum_{n=N+1}^{m} \Delta \phi_{n}\left(a_{k}+\delta_{k+1}\right) \\
&=\sum_{k=N+1}^{m}\left(\phi_{k+1}-\phi_{N+1}\right)\left(a_{k}+\delta_{k+1}\right)+\left(\phi_{m+1}-\phi_{N+1}\right) \sum_{k=m+1}^{\infty}\left(a_{k}+\delta_{k+1}\right) \\
& \leqq \\
& k=\sum_{N+1}^{\infty} \phi_{k+1}\left(a_{k}+\delta_{k+1}\right),
\end{aligned}
$$

and so $\Sigma A_{n} \Delta \phi_{n}$ converges because both $\Sigma \phi_{n+1} a_{n}$ and $\Sigma \phi_{n} \delta_{n}$ converge.

\section{Proofs of Theorems 5 and 6}

Since the proofs of these theorems are very similar, we prove only Theorem 6. We write $c_{k}(x)=1-\cos \left(k+\frac{1}{2}\right) x$. Then we obtain

$$
\begin{aligned}
-2 \sin \frac{1}{2} x \sum_{1}^{n} \lambda_{k} \sin k x & =\sum_{1}^{n} \lambda_{k}\left\{\cos \left(k+\frac{1}{2}\right) x-\cos \left(k-\frac{1}{2}\right) x\right\} \\
& =\sum_{1}^{n} \lambda_{k}\left\{c_{k-1}(x)-c_{k}(x)\right\} \\
& =\lambda_{1} c_{0}(x)-\lambda_{n} c_{n}(x)+{ }_{1}^{n-1}\left(\lambda_{k+1}-\lambda_{k}\right) c_{k}(x) .
\end{aligned}
$$

Since $\lambda_{n} \rightarrow 0$ as $n \rightarrow \infty$, we have

$$
\sum_{1}^{\infty} \lambda_{n} \sin n x=\frac{1}{2} \operatorname{cosec} \frac{1}{2} x\left\{-\lambda_{1} c_{0}(x)+\sum_{1}^{\infty}\left(\Delta \lambda_{n}\right) c_{n}(x)\right\}
$$

whenever the right-hand side is convergent.

Now, since $\Sigma \phi_{n} \delta_{n}$ and $\Sigma \lambda_{n} \Delta \phi_{n}$ converge, it follows by Theorem 2 that $\Sigma \phi_{n+1} \Delta \lambda_{n}$ is absolutely convergent. Thus $\Sigma \Delta \lambda_{n}$ is absolutely convergent and, as $\left|c_{k}(x)\right| \leqq 2$, (3.1) shows that the series $\Sigma \lambda_{n} \sin n x$ is uniformly convergent 
A GENERALIZATION OF QUASI-MONOTONE SEQUENCES 41

in any closed interval which excludes all integral multiples of $2 \pi$. Also, clearly the series converges to zero at integral multiples of $2 \pi$.

To show that $\eta(x) \Sigma \lambda_{n} \sin n x \in L(0, \pi)$, we see from (3.1) that

$$
\begin{aligned}
& \int_{0}^{\pi} \eta(x)\left|\sum_{1}^{\infty} \lambda_{n} \sin n x\right| d x \leqq \frac{1}{2}\left|\lambda_{1}\right| \int_{0}^{\pi} \eta(x) c_{0}(x) \operatorname{cosec} \frac{1}{2} x d x \\
& \quad+\int_{0}^{\pi} \eta(x) \sum_{1}^{\infty}\left|\Delta \lambda_{n}\right| c_{n}(x) \operatorname{cosec} \frac{1}{2} x d x \\
& =\frac{1}{2}\left|\lambda_{1}\right| \int_{0}^{\pi} \eta(x) \tan \frac{1}{4} x d x+\sum_{1}^{\infty}\left|\Delta \lambda_{n}\right| \int_{0}^{\pi} \eta(x) c_{n}(x) \operatorname{cosec} \frac{1}{2} x d x \\
& \leqq \frac{1}{4}\left|\lambda_{1}\right| \int_{0}^{\pi} x \eta(x) d x+8 \sum_{1}^{\infty}\left|\Delta \lambda_{n}\right|\left\{n^{2} \int_{0}^{1 / n} x \eta(x) d x+\int_{1 / n}^{\pi} x^{-1} \eta(x) d x\right\} .
\end{aligned}
$$

This latter quantity is finite since $x \eta(x) \in L(0, \pi)$ and $\Sigma \phi_{n}\left|\Delta \lambda_{n}\right|$ is convergent.

\section{REFERENCES}

(1) R. P. BoAs, Quasi-positive sequences and trigonometric series, Proc. London Math. Soc. (3) 14A (1965), 38-46.

(2) S. M. SHAH, A note on quasi-monotone series, Math. Student 15 (1947), 19-24.

(3) S. M. SHAH, Trigonometric series with quasi-monotone coefficients. Proc. American Math. Soc. 13 (1962), 266-273.

(4) O. SzÁsz, Quasi-monotone series, American J. Math. 70 (1948), 203-206.

Department of Mathematics, UNIVERSITY OF SURREY, LoNDON, S.W.11 